\title{
SITUACIÓN DE LA EVALUACIÓN DEL DESEMPEÑO DOCENTE EN LA URACCAN RECINTO LAS MINAS, 2007-2008
}

Noel Altamirano Lumbi ${ }^{[10]}$

Iris del Rosario López Aburto ${ }^{[1]}$ Maribel Duriéz González ${ }^{[12]}$

\section{Resumen}

El presente estudio se enfocó a la evaluación del desempeño del personal docente en el Recinto Universitario con el objeto de valorar la situación actual de la evaluación del desempeño de docentes de URACCAN, Recinto Las Minas, período 2007-2008.

La metodología fue de carácter cualitativa y se asumió el estudio de caso como enfoque en el uso de técnicas como la entrevista cualitativa semiestructurada y el análisis documental.

Según los hallazgos el sistema actual de evaluación del desempeño docente es un proceso sistemático, cuya finalidad es mejorar la calidad de la enseñanza. Este proceso se efectúa cada semestre del año académico con los métodos de autoevaluación del docente, la evaluación del estudiantado y la supervisión de quienes realizan la coordinación.

Los resultados se entregan a cada docente para que vean en qué áreas tienen dominio y en cuáles necesitan mejorar. Muchas veces estos resultados no convencen a los docentes porque hacen falta técnicas válidas, fiables, objetivas e indicadores que no apuntalan de forma tal que contribuyan a mejorar la docencia, y a incrementar la autoestima, motivación y el prestigio de la docencia.

Palabras claves: Evaluación, desempeño, supervisión, autoevaluación, docencia, sistema evaluativo, planificación.

\section{Introducción}

La gestión docente, considerada la base fundamental para lograr cambios en el proceso de enseñanza-aprendizaje y de resultados que optimicen su validez profesional,

[10] MSc. Docencia universitaria. Docente URACCAN Las Minas. noealtamirano8@hotmail.com

[11] MSc. Docencia Universitaria. Administradora URACCAN Las Minas. irisdelrolopez@gmail.com

[12] Tutora. Docente maestría Docencia Universitaria. mduriez@cablenet.com.ni 
motiva inducir la evaluación del desempeño docente como uno de los elementos vitales en esta gestión.

Desde esta perspectiva la importancia de esta investigación radica en que permitirá a los líderes o responsables involucrados realizar los cambios rumbo el desempeño labor docente eficaz, darle plenitud a las expectativas de innovación profesional en la comunidad estudiantil.

Este estudio valora la situación actual del sistema evaluativo del desempeño docente, a la vez identifica elementos característicos en el análisis a las acciones que ha asumido el proceso de evaluación practicado a docentes en la URACCAN, Recitno Universitario Las Minas, Siuna, durante el período 2007-2008.

\section{Revisión de Literatura}

Elementos del proceso de evaluación del desempeño docente:

La organización docente. Se asume como otro elemento que implica el ordenamiento y la conjunción de distintos aspectos para favorecer el proceso de enseñanza-aprendizaje y propiciar calidad en la educación, donde incluye calendarización, organización de los grupos de clase, horarios y registro de estudiantes, entre otros, con el fin de alcanzar las mejores condiciones en el aprendizaje (Amarante, 2000, p.31).

La Evaluación docente. Se asumió como una teoría consignada por Crespí Jaume, (s.f), como una función social que es permanentemente valorada por sus alumnos, colegas, supervisores y todas aquellas personas que de una u otra forma son perceptoras de sus acciones.

Para Chiroque (2006), la evaluación de los docentes en servicio tiene como finalidad la apreciación justa de su grado de preparación y de sus méritos y deméritos, así como de las aptitudes demostradas en el ejercicio de la actividad magisterial.

Para Ander-Egg, (1996) unos de los elementos importante son los Propósitos de la evaluación que desde el punto de vista constructivista, se resumen en ayudar al crecimiento personal de los educandos; valorar el rendimiento del educando; detectar las dificultades de aprendizaje y los fallos que existen en el modo de enseñar y los procedimientos pedagógicos utilizados de cara a mejorar el proceso educativo y corregir, modificar o confirmar el mismo currículum y los procedimientos y estrategias pedagógicas utilizadas.

Sin embargo Engel, S (s.f), establece que los propósitos de la evaluación se enmarcan en estimular y favorecer el interés por el desarrollo profesional; Contribuir 
al mejoramiento pedagógico de las instituciones; Mejorar su modo de enseñar, sus funciones como gestor de aprendizajes y como orientador de jóvenes y sus relaciones con colegas.

Bixio (2004) plantea que la evaluación docente está referida a la evaluación de su planificación, de sus estrategias didácticas que utiliza para ayudarles a sus alumnos a aprender, de los materiales que selecciona, de las actividades a partir de las cuales propone los aprendizajes.

Para Ponce (s.f), la evaluación al personal docente, se relacionan con los diversos criterios generales y específicos para la evaluación. El dominio de los procesos de enseñanza y aprendizaje se estima de mayor importancia que cualquier otro criterio y recibe alrededor de dos terceras partes del valor de la evaluación.

Valdez (2000), plantea que las valoraciones y opiniones que se producen sobre el comportamiento o competencia de los docentes pueden dar lugar a situaciones de ambigüedad, a contradicciones, un alto nivel de subjetivismo, insatisfacción y desmotivación de los docentes así como promover recelos, miedos y rechazos.

Acciones desarrolladas en el proceso evaluativo del desempeño docente. Se asumió que la supervisión docente es una acción que implica el proceso de planificación que conlleva a un monitoreo de las actividades programadas por el docente en el aula de clase, donde un coordinador conduce las actividades de seguimiento con fines de hacer los ajustes necesarios (Harf, y Azzerboni, 2008, p. 81).

Periodicidad de las evaluaciones al desempeño docente. Es otra acción dentro de un proceso de evaluación a los docentes que según el CINDA (1997), se aplica al término de cada trimestre lo cual permite que el profesor aplique las enmiendas del caso. De la misma manera Engel (s.f), refiere que la evaluación de los estudiantes al docente, se realiza al término de cada trimestre.

\section{Materiales y Métodos}

La investigación tiene un enfoque metodológico hacia el estudio de caso, cualitativo y descriptivo. La población objeto de estudio estuvo conformada por 12 docentes de tiempo completo, 3 coordinadores de carrera y la secretaria académica del Recinto. Las técnicas utilizadas fueron el análisis documental y las entrevistas cualitativas. 


\section{Resultados y Discusión}

\section{Elementos del proceso de evaluación del desempeño docente}

Uno de los elementos del proceso de evaluación es la Planificación docente se desarrolla por medio de un proceso donde los coordinadores y la secretaria académica se reúnen a finales de cada año para discutir la organización docente y la carga académica por docente cada próximo año. Con esta propuesta la secretaria académica del recinto se traslada a las oficinas centrales de URACCAN para reunirse con la secretaria académica general quienes confirman la organización completa de todo el quehacer de la URACCAN.

En esta organización se establecen la oferta académica, el calendario académico y las diferentes actividades a desarrollar lo que se relaciona con lo que plantea Amarante, (2000), que la organización docente incluye calendarización, organización de los grupos de clase, horarios, entre otras actividades a desarrollar.

Posteriormente la secretaria académica, regresa al recinto para comunicar las acciones a desarrollar y continúa el proceso con un conjunto de actividades articuladas a fin de alcanzar lo que se desea obtener. Esto coincide también con lo que plantea Ander-Egg, (1996), que la planificación docente es un conjunto de procedimientos y acciones articuladas con el propósito alcanzar una situación elegida como deseable.

\section{Evaluación docente}

Al respecto, hubo varias connotaciones pero todos llegaron a expresar la misma idea, de tal manera que en el recinto Las Minas se interpreta como una tarea compleja, que se realiza junto con los estudiantes, el profesor y supervisores a fin de percibir algunas acciones que no están bien en función del proceso de enseñanza, esto coincide con Crespí Jaume (s.f), al referir que ésta es una función social valorada permanentemente por sus alumnos, colegas, supervisores y aquellas personas que de una $\mathrm{u}$ otra forma son perceptoras de sus acciones. También se definía como una forma de apreciar con equidad y justicia el trabajo que hacen los docentes, y reconocer a cada quien lo que realmente se merece lo que es similar a lo que plantea Chiroque, (2006), la evaluación de los docentes tiene como finalidad la apreciación justa de su grado de preparación y de sus méritos y deméritos, como de las aptitudes demostradas en la actividad magisterial.

\section{Propósito de la evaluación docente}

Los informantes, coincidieron en que el objetivo es mejorar la calidad de la enseñanzaaprendizaje a través de la mejora de la docencia. Al respecto Ander-Egg (1996), y Engel (s/f) refieren que los propósitos son: favorecer el desarrollo profesional; detectar las 
dificultades; mejorar sus capacidades pedagógicas y modo de enseñar de cara a mejorar el proceso educativo.

\section{Aspectos y criterios de evaluación al docente}

Las fuentes coincidieron y resumieron aspectos como metodología, dominio del tema y de grupo, forma de evaluación, coherencia entre materiales didácticos, el tema y las actividades que desarrollan en clase lo que coincide con Bixio (2004), al plantear que la evaluación docente está referida a la evaluación de su planificación, de sus estrategias didácticas, de los materiales que selecciona y las actividades a partir de las cuales propone los aprendizajes.

$\mathrm{Al}$ revisar los documentos de evaluación, se encontraron otros aspectos coincidentes con otras fuentes. Sin embargo, en las guías de evaluación existen aspectos referidos a planificación, actuación y evaluación. En la parte de planificación, se evalúan criterios referentes a que si el docente plasma un guión de lo que va hacer en clase, establece objetivos y contenidos o sea se refiere a la planificación de la clase. En Actuación se le evalúan criterios referidos a si el docente informa el plan del curso, motiva y fomenta el aprendizaje, toma en cuenta conocimientos previos, comunican objetivos y relación con los contenidos, presentan ejemplos, dirige la mirada, responde con precisión, es respetuoso, hace resumen al concluir la clase etc.

Con respecto al apartado de evaluación, se ve si esta se hace en base a objetivos, contenidos y actividades, si orientan los criterios, formas y momentos de evaluación, tipos de prueba a realizar, si comenta los resultados de las evaluaciones y orienta como mejorar los resultados.

Se nota que muchos de los aspectos y criterios de evaluación, se relacionan unos con otros, a lo que Ponce (s.f) refiere, que la evaluación del personal docente, relaciona diversos criterios generales y específicos. Al parecer muchos aspectos se repiten y sólo abarcan la parte pedagógica, faltarían aspectos de mayor relevancia para que la evaluación sea más integral.

\section{Métodos de evaluación docente}

Este es otro elemento compuesto por tres métodos, dentro de los cuales se encuentran: la evaluación de los estudiantes, es una guía con un cuestionario para que los estudiantes opinen sobre los aspectos de planificación, actuación y evaluación esto coincide con Engel (s.f), esta evaluación, se hace a través de una encuesta con los alumnos respecto de su satisfacción con los resultados obtenidos y la actuación del docente. 


\section{El método de autoevaluación}

Los docentes establecen la forma en que han cumplido los distintos aspectos de evaluación, lo que también coincide con Engel (s.f), al referir que es una narrativa, donde el profesor analiza como cumple con cada uno de los criterios de evaluación. Mientras que la supervisión o la visita al salón de clases es donde los coordinadores observan aspectos didácticos y metodológicos tanto a los docentes horarios como de tiempo completo.

\section{Opinión de docentes acerca de los evaluadores}

Este es un elemento donde la opinión más común en cuanto a los docentes quienes expresan que la evaluación de los estudiantes es subjetiva y parcializada, señalan aspectos que no son creíbles y van en detrimento de los docentes. Estas evaluaciones están influenciadas por factores personales, lo cual coincide con Engel, (s.f), al decir, que este tipo de evaluación puede estar influida por un sinnúmero de factores subjetivos.

Otras opiniones establecen referencias a que los evaluadores no son especialistas para dar un buen dictamen del docente, puesto que son personas con conocimientos generales pedagógicos pero no reciben asistencia de alguien especializado. Según Ponce (s.f), la evaluación de los académicos debiera estar a cargo de consejos técnicos con el apoyo de comisiones facultativas, mientras que Villalobos, (2008) expresa que esta debiera de ser ejercida por personal cualificado que la eleve al sitial que le corresponde.

\section{Niveles de satisfacción}

La satisfacción docente con respecto a los resultados de las evaluaciones, se pudo constatar que no se encuentran conformes con los resultados de las evaluaciones que se les practica por considerarlas influidas por un sinnúmero de factores personales, y muchos de los aspectos negativos que les señalan los dejan dudando, hasta cierto puntos extrañados y que muchas veces no se sienten satisfechos con respecto a los indicadores evaluados.

\section{Fortalezas y limitaciones de las evaluaciones al docente}

Las fortalezas del sistema actual de evaluación es que permite documentar al docente sobre lo que se debe mejorar; Es flexible puesto que los coordinadores pueden ser apoyados por docentes que tienen competencia y dominio sobre alguna materia; reduce las actuaciones pedagógicas incorrectas en que incurren los docentes. Las limitaciones refieren que se señalan los problemas sin dar respuesta con las capacitaciones; no dan un resultado creíble y confiable de la realidad del desempeño docente puesto que es difícil triangular la información; los instrumentos de evaluación contienen demasiada información. 


\section{Acciones desarrolladas en el proceso evaluativo del desempeño docente}

Una de las acciones desarrollas es la forma de planificar la evaluación al desempeño docente para la cual los coordinadores trazan un programa de acompañamiento semestral y se lo pasan a la secretaría académica para que lo apruebe. Con el programa, le dan seguimiento a las actividades programadas por el docente en su syllabus, a lo que Harf, y Azzerboni (2008) señalan que la supervisión docente es un proceso de monitoreo de las actividades programadas por el docente, donde un coordinador conduce las actividades de seguimiento a fin de hacer los ajustes necesarios.

Otra acción desarrollada por quienes coordinan, es aplicar las evaluaciones que por lo general se hacen con una frecuencia semestral, así la evaluación de los estudiantes al docente se realiza al final de cada semestre, esto difiere con el CINDA, (1997), porque refiere que la evaluación de los docentes por los alumnos, se aplica al término de cada trimestre. La autoevaluación del docente también es semestral, mientras que la supervisión puede ser al inicio o intermedio del semestre.

Otra de las acciones realizadas por los coordinadores es un consolidado de las evaluaciones (estudiantes, coordinadores y autoevaluaciones), y se lo notifican a cada uno de los docentes, quienes firman aceptando que el informe es real o que están de acuerdo con lo que se les informa, adquiriendo el compromiso de mejorar en el próximo semestre sobre aquellos aspectos en los que se ven afectados. Al respecto Valdés, (2000) señala que el maestro conoce con precisión cómo es percibido su trabajo cuando recibe un informe de los aspectos más y menos logrados de su desempeño, según la información proporcionada por los instrumentos de evaluación.

\section{Propuesta de un modelo de evaluación y estímulo al desempeño del personal docente de la URACCAN, Recinto Las Minas}

Este modelo propone concebir la evaluación como un proceso sistemático que consiste en la aplicación de normas y procedimientos que permite el acompañamiento al desempeño de la planta docente. Que el objetivo sea identificar logros y apoyar a las áreas que necesitan mejorar y así contribuir a la promoción y estimulo de la docencia.

Que los encargados de la evaluación sean una Comisión Técnica creada al principio de cada año integrada por un coordinador o coordinadora, secretaria académica, con el apoyo de un experto invitado o docentes colegas que tengan conocimientos sobre la asignatura que se facilita.

\section{Que los aspectos de la evaluación con sus respectivos criterios sean:}

Capacidades pedagógicas y dominio técnico de su asignatura, los criterios son: dominio de la materia. Explica y comunica con claridad. Recapitulación de clase anterior y 
comunicación de contendidos, metodología y objetivos. Motiva a los estudiantes. Escribe en la pizarra con claridad de ideas y legibilidad. Utiliza metodologías alternas. Atiende individualmente a los estudiantes. Hace participativa la clase. La evaluación es coherente con objetivos y contenidos. Concluye recapitulando lo más importante y orientando actividades de aprendizaje.

Relaciones interpersonales con los estudiantes y comunidad universitaria en general: Se preocupa y comprende problemas de sus estudiantes. Posibilita expectativas de aprendizaje. Es flexible para aceptar diversidad de opinione. Crea un ambiente para que el estudiantado conozca sus derechos y responsabilidades.

Actitudes personales y emocionales para desarrollar su trabajo: Posee el docente vocación pedagógica. Refleja Autoestima en su comportamiento. Manifiesta satisfacción con la labor que realiza.

Prácticas de valores interculturalidad y género: Practica valores como equidad, honestidad, transparencia y creatividad. Promueve y promociona la práctica de la interculturalidad. Promueve y desarrolla acciones de género.

Responsabilidad y grado de cumplimiento en el desempeño de sus funciones profesionales: Planifica y prepara syllabus y unidades didácticas de sus asignaturas. Llega al salón de clases puntualmente. Orienta a los estudiantes en los trabajos independientes. Desempeña con responsabilidad y eficiencia las funciones correspondientes a su cargo.

Actualización profesional y pedagógica: Participa en eventos de adiestramiento pedagógicos y demás actividades de capacitación a fin de mejorar su desarrollo personal y profesional. Maneja información actualizada sobre la materia y su profesión. Elabora y utiliza documentos didácticos pertinentes basados en la investigación como parte integral del quehacer docente.

Disciplina laboral: Cumple con la jornada y horario de trabajo establecido. Realiza sus labores y funciones en el modo y tiempo convenido. Muestra una conducta respetuosa con la institución, autoridades y demás colegas. Conserva y utiliza los documentos, materiales y demás bienes institucionales de forma cuidadosa.

Se propone que los instrumentos de evaluación con que se realicen las evaluaciones del desempeño docente sea por medio de la autoevaluación; el portafolio como una opción si no desea la autoevaluación narrativa; evaluación por acompañamiento en el salón de clases; evaluación en la opinión de los estudiantes y un Informe escrito de los coordinadores. 
También se propone que los resultados de las evaluaciones sean firmados por los miembros de la Comisión Técnica, y en un plazo de quince días de concluida la evaluación notifiquen al docente el resultado de la misma y se elabore un plan de capacitación individual a implementarse en los siguientes tres meses.

Por último se propone que los docentes sean incentivados, ya sea con estímulos económicos, materiales, recreativos o exclusivos como un pergamino u otro galardón que consideren, pero también que docentes reincidentes se sancionen excluyéndolos de cualquier capacitación o excursión recreativa que se realice u otra que considere la comisión a excepción del despido a no ser que lo amerite.

Se propone que los formatos de evaluación contengan la siguiente estructura:

Objetivo Asignatura: Fecha: Docente:

\begin{tabular}{lllll}
\hline Aspectos & \multicolumn{1}{c}{ Indicadores } & $\begin{array}{c}\text { Desempeño } \\
\text { alcanzado }\end{array}$ & $\begin{array}{l}\text { Desempeño } \\
\text { en progreso }\end{array}$ & $\begin{array}{l}\text { Desempeño } \\
\text { sin progreso }\end{array}$ \\
\hline \multirow{3}{*}{$\begin{array}{l}\text { Capacidad } \\
\text { pedagógica }\end{array}$} & $\begin{array}{l}\text { 1. Dominio de la materia } \\
\text { que enseña. }\end{array}$ & & \\
\cline { 2 - 5 } & $\begin{array}{l}\text { 2. Explica y comunica } \\
\text { con claridad. }\end{array}$ & & \\
\hline
\end{tabular}

El portafolio deberá contener:

- Portada donde en la parte superior diga Portafolio; parte media carpeta de trabajo y el nombre de la asignatura, y en la parte de abajo que aparezca el nombre del docente, el semestre y año.

- Página de identificación e información del docente, respecto de su filosofía, experiencia profesional o aspiraciones, inclusive identificación con otro ser por ejemplo:

\section{Diario del desempeño profesional}

A partir de aquí le proponemos un diario de desempeño profesional por aspectos donde se evidencien sus habilidades académicas: 
Me identifico con un:

Por ser hábil en lo que hace.

Es el ave regional de la Costa.

Gorrión

Mantiene un equilibrio ideal.

No descuida su planeo.

Su aspecto no ofende.

Tiene un dulce vivir.

Y luego, establecer una serie de evidencias referidas a:

Capacidades pedagógicas y dominio de asignaturas; relaciones interpersonales

con los estudiantes y universidad en general; actitudes personales positivas y

emocionales para desarrollar su trabajo; responsabilidad en el desempeño; actua-

lización profesional y pedagógica.

\section{Conclusiones}

El sistema actual de evaluación al desempeño docente se caracteriza por evaluar los

Aspectos de actuación, planificación y evaluación semestralmente a través de los instrumentos de autoevaluación, evaluación de los estudiantes a los docentes y supervisión directa de los coordinadores en el aula.

Las acciones desarrolladas en el proceso de evaluación por parte de los coordinadores, están orientadas a programar las supervisiones y evaluaciones que les efectuaran a los docentes semestralmente y a la consolidación de los resultados de las evaluaciones que posteriormente se le informarán a los docentes.

La propuesta del nuevo modelo de evaluación al desempeño docente incorpora combinadamente los métodos de autoevaluación, evaluación estudiantil al personal docente, supervisión por parte de los coordinadores en el aula de clase y portafolio, de tal manera que permiten realizar una evaluación al desempeño docente más integral, motivador y de superación profesional.

\section{Lista de Referencias}

Amarante, A. María. (2000). Gestión Directiva Módulos 1 a 4. Buenos Aires Argentina: Editorial Magisterio del Rio de la Plata.

Ander-Egg, Ezequiel. (1996). La planificación educativa. Conceptos, estrategias y técnicas para educadores. Buenos Aires: Magisterio del Río de la Plata. 
Bixio, Cecilia (2004). Como planificar y evaluar en el aula: propuesta y ejemplos. Santa Fe Argentina: Homo Sapiens. Segunda edición.

CINDA, (1997). Gestión docente universitaria. Modelos comparados. Santiago, Chile: América Latina Formación Académica.

Harf, Ruth y Azzerboni, Delia. (2008). Estrategias para la acción directiva: Condiciones para la gestión curricular y el acompañamiento pedagógico. Buenos Aires Argentina: Centro de Publicaciones Educativas.

\section{Recursos Electrónicos}

Chiroque Chunga, Sigfredo (2006 Enero). Evaluación de desempeños docentes. [En línea]. Disponible en: www.educared.edu.pe/modulo/upload/96723594.pdf

Crespí Jaume, María de la S (s.f). La Evaluación del Desempeño Docente: un espacio abierto para el desarrollo profesional del educador. [En línea]. Disponible en: www.contexto-educativo.com.ar

Engel S, (s.f). ¿Cómo evaluar el Desempeño Docente? [En línea]. Disponible en: www. monografias.com/trabajos17/evaluar-docentes/evaluar-docentes.shtml

Ponce, (s.f), Guías para la evaluación del personal docente. [en línea]. en: ponce.inter. $\mathrm{edu} / \mathrm{nhp} /$ contents/RecHumanos-GuiasEvalPersonalDocente.htm

Valdés Veloz (2000). Encuentro Iberoamericano sobre Evaluación del Desempeño Docente. Ciudad de México. [En línea]. Disponible en www.oei.es/de/rifado1.htm

Villalobos, (2008). La supervisión como apoyo al docente [en línea]. Disponible en: remembranza.wordpress.com/2008/.../ [05-09-2009] 